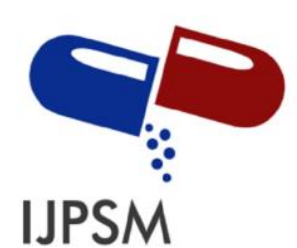

Bagmar Anjali et al, Int. Journal of Pharmaceutical Sciences and Medicine (IJPSM),

Vol.6 Issue. 9, September- 2021, pg. 79-87

ISSN: 2519-9889

Impact Factor: 3.426

\title{
SUSTAINED RELEASE MATRIX DRUG DELIVERY SYSTEM: AN OVERVIEW
}

\author{
Bagmar Anjali; Tikariya Komal \\ Swami Vivekanand College of Pharmacy, Indore (M.P) \\ DOI: 10.47760/ijpsm.2021.v06i09.006
}

\begin{abstract}
Oral delivery of drugs is the most preferable route of drug delivery due to the ease of administration, patient compliance and flexibility in formulation, etc. Sustained release constitutes are the dosage form that provides medication over an extended time or denotes that the system is able to provide some actual therapeutic control whether this is of a temporal nature, spatial nature or both. The objective of the study was to explore the necessity, advantages and various techniques of extended release matrix tablet to get a constant drug delivery rate and reproducible kinetics for advance delivery. Matrix tablets are the type of controlled drug delivery systems, which release the drug in continuous manner by dissolution controlled as well as diffusion controlled mechanisms. To control the release of the drugs, which are having different solubility properties, the drug is dispersed in swellable hydrophilic substances, an insoluble matrix of rigid non swellable hydrophobic materials or plastic materials. Matrix tablets can be formulated by either direct compression or wet granulation method by using a variety of hydrophilic or hydrophobic polymers. The extended release matrix tablets can assure better patient compliance through reduction in total dose and dosage regimen, which can be great help to treat chronic diseases. This review highlights the types of matrices, mechanisms involved and evaluation studies.
\end{abstract}

\section{INTRODUCTION}

Oral delivery of drugs is the most preferable route of drug delivery due to the ease of administration, patient compliance and flexibility in formulation, etc. Many of the drug delivery systems available in the market are oral drug delivery type systems. Approximately $50 \%$ of the drug delivery systems available in the market are oral drug delivery systems. For the release of medication for a long period of time after administration of a single dose. Sustained release Matrix tablets are used. Theoretically and desirably a sustained release delivery device, should release the drug by a zero-order process which would result in a blood-level time profile similar to that after 


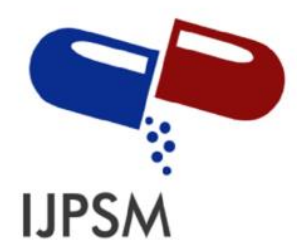

Bagmar Anjali et al, Int. Journal of Pharmaceutical Sciences and Medicine (IJPSM), Vol.6 Issue. 9, September- 2021, pg. 79-87

ISSN: 2519-9889

Impact Factor: 3.426

intravenous constant rate infusion. The majority of oral sustained release systems rely on dissolution, diffusion or a combination of both mechanisms, to generate slow release of drug to the gastrointestinal tract. Plasma drug concentration-profiles for conventional tablet or capsule formulation, a sustained release formulation, and a zero order sustained release formulation.

Sustained release constitutes are the dosage form that provides medication over an extended time or denotes that the system is able to provide some actual therapeutic control whether this is of a temporal nature, spatial nature or both. Sustained release system generally do not attain zero order type release and usually try to mimic zero order release by providing drug in a slow first order. Sustained release dosage form will provide a therapeutic concentration of the drug in the blood that is maintained throughout the dosing interval with a reduction in a peak concentration ratio.

\section{MATRIX TABLETS}

Matrix tablet are the type of controlled drug delivery systems, which release the drug in continuous manner by dissolution controlled as well as diffusion controlled mechanisms. To control the release of the drugs, which are having different solubility properties, the drug is dispersed in swellable hydrophilic substances, an insoluble matrix of rigid non swellable hydrophobic materials or plastic materials. One of the least complicated approaches to the manufacture of sustained release dosage forms involves the direct compression of blend of drug release, retardant material and additives to formulate a tablet in which the drug is embedded in a matrix of the release retardant. Alternatively drug and release retardant blend may be granulated prior to compression.

\section{ADVANTAGES OF MATRIX TABLET}

- Easy to manufacture

- Versatile, effective and low cost

- Can be made to release high molecular weight compounds

- The sustained release formulations may maintain therapeutic concentrations over prolonged periods.

- The use of sustain release formulations avoids the high blood concentration.

- Sustain release formulations have the potential to improve the patient compliance. 


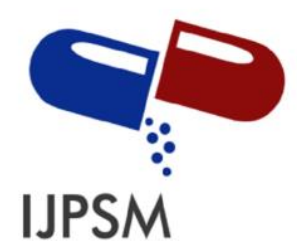

Bagmar Anjali et al, Int. Journal of Pharmaceutical Sciences and Medicine (IJPSM),

Vol.6 Issue. 9, September- 2021, pg. 79-87

ISSN: 2519-9889

Impact Factor: 3.426

- Reduce the toxicity by slowing drug absorption.

- Minimize the local and systemic side effects.

- Improvement in treatment efficacy.

- Minimize drug accumulation with chronic dosing.

- Improvement of the ability to provide special effects.

Ex: Morning relief of arthritis through bed time dosing

\section{DISADVANTAGES OF MATRIX TABLET:}

- The remaining matrix must be removed after the drug has been released.

- High cost of preparation.

- The release rates are affected by various factors such as, food and the rate transit through the gut.

The drug release rates vary with the square root of time. Release rate continuously diminishes due to an increase in diffusional resistance and/or a decrease in effective area at the diffusion front. However, a substantial sustained effect can be produced through the use of very slow release rates, which in many applications are indistinguishable from zero-order.

\section{MATRIX TABLET GENERALLY CLASSIFIED INTO DIFFERENT TYPES:}

A. Hydrophilic Matrix Tablet: Hydrophilic matrix generally used to control the release rate of drug. The matrix can be tableted by direct compression of the blend of active ingredient and certain hydrophilic carriers or from a wet granulation containing the drug and hydrophilic matrix materials.

B. Fat-wax Matrix Tablet: Various technique used for incorporation of drug into fat wax granulation which involve spray congealing in air, blend congealing in an aqueous media with or without the aid of surfactant and spray drying Technique. Bulk congealing method, a suspension of drug and melted fat wax is allowed to solidify and then comminuted for sustained-release granulations

C. Plastic Matrix Tablet (Hydrophobic matrices): Sustained release tablets based upon an inert compressed plastic matrix have been used widely. Release is usually delayed because the dissolved drug has to diffuse through capillary network between the 


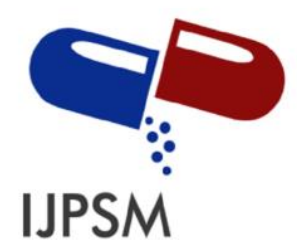

Bagmar Anjali et al, Int. Journal of Pharmaceutical Sciences and Medicine (IJPSM), Vol.6 Issue. 9, September- 2021, pg. 79-87

ISSN: 2519-9889

Impact Factor: 3.426

compacted polymer particles. Plastic matrix tablets, in which the active ingredient is embedded in a tablet with coherent and porous skeletal structure, can be easily prepared by direct compression of drug with plastic materials provided the plastic material can be comminuted or granulated to desired particle size to facilitate mixing with the drug particle.

D. Biodegradable Matrices: These consist of the polymers which comprised of monomers linked to each other by functional groups and have unstable linkage in the backbone. It is biologically degraded or eroded by enzymes generated by surrounding living cells or by non-enzymatic process into oligomers and monomers that can be metabolized or excreted. Examples are natural polymers such as proteins, polysaccharides and modified natural polymers, synthetic polymers such as aliphatic poly (esters) and poly anhydrides.

E. Mineral Matrices: Mineral matrices consist of polymers which are obtained from various species of seaweeds. Example: Alginic acid which is a hydrophilic carbohydrate obtained from species of brown seaweeds (Phaephyceae) by the use of dilute alkali.

\section{ON THE BASIS OF POROSITY OF MATRIX:}

Matrix system can also be classified according to their porosity and consequently, Macro porous; Micro porous and Non-porous systems can be identified:

- Macro porous Systems: In such systems the diffusion of drug occurs through pores of matrix, which are of size range 0.1 to $1 \mu \mathrm{m}$. This pore size is larger than diffusant molecule size.

- Micro porous System: Diffusion in this type of system occurs essentially through pores. For micro porous systems, pore size ranges between $50-200 \mathrm{~A}^{\circ}$, which is slightly larger than diffusant molecules size.

- Non-porous System: Non-porous systems have no pores and the molecules diffuse through the network meshes. In this case, only the polymeric phase exists and no pore phase is present. 


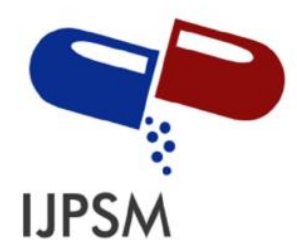

Bagmar Anjali et al, Int. Journal of Pharmaceutical Sciences and Medicine (IJPSM),

Vol.6 Issue. 9, September- 2021, pg. 79-87

ISSN: 2519-9889

Impact Factor: 3.426

\section{POLYMERS USED IN MATRIX TABLET:}

- Hydrogels: Polyhydroxyethylemethylacrylate (PHEMA), Cross-linked polyvinyl alcohol (PVA), Cross-linked polyvinyl pyrrolidone (PVP), Polyethylene oxide (PEO), Polyacrylamide (PA)

- Soluble polymers: Polyethyleneglycol (PEG), polyvinyl alcohol (PVA), Polyvinylpyrrolidone (PVP), Hydroxypropyl methyl cellulose (HPMC)

- Biodegradable polymers: Polylactic acid (PLA), Polyglycolic acid (PGA), Polycaprolactone (PCL), Polyanhydrides, Polyorthoesters

- Non-biodegradable polymers: Polyethylene vinyl acetate(PVA), Polydimethylsiloxane (PDS), Polyether urethane (PEU), Polyvinyl chloride (PVC), Cellulose acetate (CA), Ethyl cellulose (EC).

\section{MECHANISM OF DRUG RELEASE FROM THE MATRIX TABLETS:}

Drug in the outside layer exposed to the bathing solution is dissolved first and then diffuses out of the matrix. This process continues with the interface between the bathing solution and the solid drug moving toward the interior. It follows that for this system to be diffusion controlled, the rate of dissolution of drug particles within the matrix must be much faster than the diffusion rate of dissolved drug leaving the matrix.

Derivation of the mathematical model to describe this system involves the following assumptions:

1) A pseudo-steady state is maintained during drug release.

2) The diameter of the drug particles is less than the average distance of drug diffusion through the matrix.

3) The bathing solution provides sink conditions at all times.

The release behavior for the system can be mathematically described by the following equation:

$\mathrm{dM} / \mathrm{dh}=\mathrm{CO} . \mathrm{dh}-\mathrm{Cs} / 2$

Where, $\mathrm{dM}=$ Change in the amount of drug released per unit area.

$\mathrm{dh}=$ Change in the thickness of the zone of matrix that has been depleted of drug. 


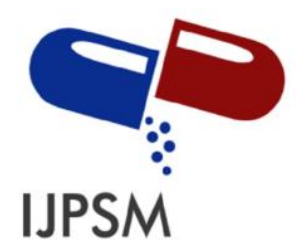

Bagmar Anjali et al, Int. Journal of Pharmaceutical Sciences and Medicine (IJPSM),

Vol.6 Issue. 9, September- 2021, pg. 79-87

ISSN: 2519-9889

Impact Factor: 3.426

$\mathrm{C} 0=$ Total amount of drug in a unit volume of matrix.

$\mathrm{Cs}=$ Saturated concentration of the drug within the matrix.

Additionally, according to diffusion theory:

$\mathrm{dM}=(\mathrm{Dm} . \mathrm{Cs} / \mathrm{h}) \mathrm{dt}$.

Where, $\mathrm{Dm}=$ Diffusion coefficient in the

matrix.

$\mathrm{h}=$ Thickness of the drug-depleted

matrix

$\mathrm{dt}=$ Change in time.

By combining equation (i) and equation (ii) and integrating:

$M=[C s . \operatorname{Dm}(2 \mathrm{Co}-\mathrm{Cs}) \mathrm{t}] \mathbf{1 / 2}$

When the amount of drug is in excess of the saturation concentration then:

$M=[2 C s . D m . C 0 . t] 1 / 2$

Equation (iii) and eq. (iv) relate the amount of drug release to the square-root of time. Therefore, if a system is predominantly diffusion controlled, then it is expected that a plot of the drug release vs. square root of time will result in a straight line. Drug release from a porous monolithic matrix involves the simultaneous penetration of surrounding liquid, dissolution of drug and leaching out of the drug through tortuous interstitial channels and pores. The volume and length of the openings must be accounted for in the drug release from a porous or granular matrix:

$M=[D s . C a . p / T .(2 C o-p . C a) t] 1 / 2$

Where, $\mathrm{p}=$ Porosity of the

matrix

$\mathrm{t}=$ Tortuosity

$\mathrm{Ca}=$ solubility of the drug in the release medium 


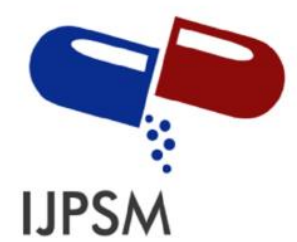

Bagmar Anjali et al, Int. Journal of Pharmaceutical Sciences and Medicine (IJPSM),

Vol.6 Issue. 9, September- 2021, pg. 79-87

ISSN: 2519-9889

Impact Factor: 3.426

Ds $=$ Diffusion coefficient in the release medium.

$\mathrm{T}=$ Diffusional pathlength

For pseudo steady state, the equation can be written as:

$M=[2 D . C a . C 0(p / T) t] ~ 1 / 2$

The total porosity of the matrix can be calculated with the following equation:

$$
\mathbf{p}=\mathbf{p a}+\mathbf{C a} / \mathbf{\rho}+\mathrm{Cex} / \rho \mathrm{ex} \ldots \ldots \ldots \ldots(\text { vii) }
$$

Where, $\mathrm{p}=$ porosity

$$
\begin{gathered}
\rho=\text { Drug density } \\
\text { pa }=\text { Porosity due to air pockets in the matrix } \\
\rho \mathrm{ex}=\text { Density of the water soluble excipients } \\
\text { Cex }=\text { Concentration of water soluble excipients }
\end{gathered}
$$

For the purpose of data treatment, equation (vii) can be reduced to:

$M=k \cdot t$ 1/2

Where, $\mathrm{k}=$ constant.

So the amount of drug released versus the square root of time will be linear, if the release of drug from matrix is diffusion controlled.

\section{EVALUATION OF ORAL SUSTAINED RELEASE TABLETS}

- Thickness of tablet: Thickness of tablet is evaluated by using micrometer screw gauge. Test is carried out randomly on twenty tablets and average values are calculated.

- Hardness of tablet: Hardness of tablet of each batch is evaluated by monsantto hardness tester and average values are calculated.

- Uniformity of weight: 20 tablets are selected randomly and weighed individually and collectively; average weight is calculated. 


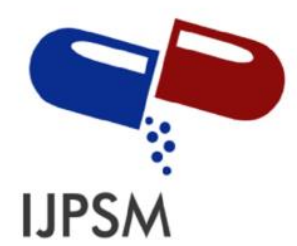

Bagmar Anjali et al, Int. Journal of Pharmaceutical Sciences and Medicine (IJPSM),

Vol.6 Issue. 9, September- 2021, pg. 79-87

ISSN: 2519-9889

Impact Factor: 3.426

$\%$ of weight variation $=($ Individual Weight Average weight/Average Weight $) \times 100$

- Uniformity of content: This test is done to make sure that every tablet should contain the same amount of active ingredient with little or no variation within a batch. For content uniformity test 30 tablets are selected and 10 are assayed individually. At least 9 must assay between $\pm 15 \%$ of the declared potency and should not exceed $\pm 25 \%$

- Friability: 20 tablets are weighed and placed in fribilator. The chamber is rotated for 4 minutes at a speed of 25 r.p.m. the tablets are removed from the chamber and weighed again. Loss in weight indicates friability. The tablets to be considered of good quality if loss in weight is less than $0.8 \%$

- In vitro dissolution studies: The test is carried out to measure the amount of time required for certain percentage of drug to go into the solution under the specific test conditions. Rotating paddle type and rotating basket type apparatus can be used as per pharmacopoeial standards or as mentioned in monograph of particular drug42. The test is passed if for each of the five tablets, the amount of active ingredient in solution is not less than $70 \%$ of the stated amount or as specified in the monograph of the API in pharmacopoeia

\section{CONCLUSION:}

The review of the article is focused on the formulation and uses of sustained released matrix type drug delivery system. By the above discussion, it can be easily concluded that sustained-release formulation are helpful in increasing the efficiency of the dose as well as they are also improving the patient's compatibility matrix forming polymers can be successfully used to prepare Matrix tablets, releasing drug in a controlled manner. Preparatory procedures easily allow adaptation of release kinetics to delivery needs. This suitability of matrix forming polymers, to various drug delivery systems preparation confirms the importance of these specialized excipients in pharmaceutical application. So, this extend release formulation can be used for a suitable formulations for arthritis or diabetes. The drugs can be incorporated and better pharmacological action can be achieved. This article may be beneficial for many researcher interested to work on the matrix tablets. 


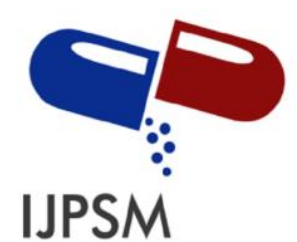

Bagmar Anjali et al, Int. Journal of Pharmaceutical Sciences and Medicine (IJPSM), Vol.6 Issue. 9, September- 2021, pg. 79-87

ISSN: 2519-9889

Impact Factor: 3.426

\section{References}

[1]. YW. Oral drug delivery systems in novel drug delivery pharmaceutical technology. Marcel Dekker Inc. New York. 1992;139-52.

[2]. Qiu Y, Zhang G. Research and development aspects of oral controlled release dosage forms. Handbook of pharmaceutical controlled release technology. 1st Indian Ed. Replika press. New York. 2005;465-503.

[3]. Chien YW. Oral drug delivery systems in novel drug delivery pharmaceutical technology. Marcel Dekker Inc. New York. Basel. 1992;152-96.

[4]. Chen X, Wen H, Park K. Challenges and new technologies of oral controlled release. Oral Controlled Release Formulation Design and Drug Delivery: Theory to Practice. 2010;257-77.

[5]. Ali J, Khar RK, Ahuja A. A Textbook of Biopharmaceutics \& Pharmacokinetics. Birla Publications Pvt. Ltd. Delhi. 2008;252-72.

[6]. Agarwal G, Kaushik A. Pharmaceutical Technology-II. 1st Ed. CBS Publishers, New Delhi. 2012;12334,174-89.

[7]. Brahmankar DM, Jaiswal SB. Controlled release medication. Biopharmaceutics and Pharmacokinetics- A treatise. 2nd Ed. Vallabh Prakashan. Delhi. 2009;397-400.

[8]. Zalte HD, Saudagar RB. Review on sustained release matrix tablet. Int J Pharm Biol Sci. 2013;3(4):17-29.

[9]. Ratnaparkhi MP, Gupta JP. Sustained release drug delivery system- An overview. Int J Pharma Res Rev. 2013;2(3):11-21.

[10]. Tapaswi RD, Verma P. Matrix tablets: An approach towards oral extended release drug delivery. Int J Pharma Res Rev. 2013; 2(2):12-24.

[11].Patel H, Panchal DR, Patel U, et al. Matrix type drug delivery system: A Review. : J Pharm Sci Bio-Sci Res. 2011;1(3):143-51. 